\title{
Semantic Scheduling of Virtualized Infrastructures for Scientific Workflows
}

\author{
Idafen Santana-Pérez \\ Ontology Engineering Group - UPM \\ Email: isantana@fi.upm.es \\ Address: Avda. Montepríncipe, s/n \\ Boadilla del Monte, 28660, España \\ Dissertation Advisor: María S. Pérez-Hernández \\ DOCTORAL DISSERTATION COLLOQUIUM \\ EXTENDED ABSTRACT
}

Keywords-Cloud Computing \& Architectures, Partitioning, Mapping, and Scheduling, Virtualization and Virtual Machines

\begin{abstract}
Virtualized Infrastructures are a promising way for providing flexible and dynamic computing solutions for resourceconsuming tasks. Scientific Workflows are one of these kind of tasks, as they need a large amount of computational resources during certain periods of time.
\end{abstract}

To provide the best infrastructure configuration for a workflow it is necessary to explore as many providers as possible taking into account different criteria like Quality of Service, pricing, response time, network latency, etc. Moreover, each one of these new resources must be tuned to provide the tools and dependencies required by each of the steps of the workflow.

Working with different infrastructure providers, either public or private using their own concepts and terms, and with a set of heterogeneous applications requires a framework for integrating all the information about these elements. This work proposes semantic technologies for describing and integrating all the information about the different components of the overall system and a set of policies created by the user. Based on this information a scheduling process will be performed to generate an infrastructure configuration defining the set of virtual machines that must be run and the tools that must be deployed on them.

\section{RELATED WORK}

One of the main problems of providing virtualized infrastructures by combining several providers is their heterogeneity. Semantics have been proposed and used as a solution for dealing with heterogeneous resources in the grid field and nowadays there are several initiatives that work with semantic technologies applied to cloud federations problems. An important contribution to the state of the art in this area is described in [1] and [2].

Another work done in this field is exposed in [3], where an ontology-based discovery system is used to fill the gap between user and provider's notation. To achieve this, they translate user requirements and virtual appliances to the well known Open Virtualization Format (OVF) [4] to filter which virtual appliances fulfill those requirements. An interesting initiative in this area is the mOSAIC [5] project which uses, as we propose in this work, a network of ontologies and a set of APIs for solving federated clouds interoperability. However those ontologies focus on describing providers, leaving applications and workflows description out of their scope.

Regarding to the on-demand infrastructure configuration (explained in section III) two interesting contributions have been recently announced by one of the most relevant IaaS providers worldwide, Amazon Web Services [6]: (i) AWS Marketplace [7], an online store in which software vendors expose their products so users can find, buy and automatically deploy this applications in their AWS instances; (ii)AWS Simple Workflow Service [8], a fully managed workflow service for building scalable, resilient applications, allowing users to define a set of tasks and deploy them in the AWS SWF engine to be executed coordinately.

Nowadays there are several workflow management systems and engines providing a set of tools for executing workflows in Grid and Cloud infrastructures. Some of the most relevant in the scientific area are Pegasus [9] and Taverna [10]. Even though they implement methods to map workflow tasks to computational resources belonging to their infrastructures and execute them, they do not provide ways for generating custom infrastructure configuration and therefore they can not provide an optimal and dynamic solution.

Semantics also has been used in the context of scientific workflows to describe them. One of the most relevant approaches in this area is Wings [11], a semantic workflow system that allows to define scientific computational experiments by using semantics, allowing to create and validate scientific workflows. The semantic representation used in Wings does not allow to describe the software components and the depen- 
dencies involved in the execution of the computational tasks.

\section{SCIENTIFIC WORKFLOWS}

For the purpose of this work we consider Scientific Workflows as a set of tasks that must be performed coordinately in a set of computational resources guaranteeing a certain quality of service. These tasks need each of them a certain configuration, that is, a set of hardware features and software stack enabling the task to be performed.

Reproducibility is a key aspect of scientific experiments and hence of scientific workflows. Usually workflow reproducibility is defined in terms of input and output data, parameters and activities, without taking into account the infrastructure for executing it. Adding information about hardware and software requirements would increase the reproducibility of the workflow, allowing to reproduce the same infrastructure, or a very similar one, to the one used in the original experiment.

\section{ON-DEMAND INFRASTRUCTURE CONFIGURATION}

Handling different types of experiments implies working with several kind of processes, using different tools each one of them. A naive approach to support the execution of the workflow could be to have a physical infrastructure with all these tools installed. Doing so may lead to several problems, like having two or more instances of the same kind of tool (e.g. database engine) running on the same machine, different versions of the same tool (e.g. Java VM) or configuration parameters collision (e.g. two or more web services using the same port).

Using virtualization techniques allows us to get rid of the need of having a physical fixed infrastructure, increasing the dynamicity of the solution. Instead of having a physical machine with the whole software stack installed on it we could have a set of virtual machine images with all the possible combination of tools deployed on it. Even though it is a valid solution it implies a large number of virtual images to be stored and managed, which leads to a high costly solution in terms of storage and even money (in the case of public providers).

In our approach we want to create a virtual machine fulfilling hardware requirements for a given task and try to dynamically deploy only the necessary tools to execute that task on it.

\section{ARChitecture}

To implement the ideas exposed in this work we propose an architecture (depicted in figure 1) in which we define a set of components to integrate several virtual infrastructure providers. The main component of this architecture is the Scheduler which is responsible for defining the set of virtual machines to be created and the tools that must be deployed on it, based on the information retrieved from the Information System and the Catalog (which contains the information about virtual machine images and applications). The Adapters are

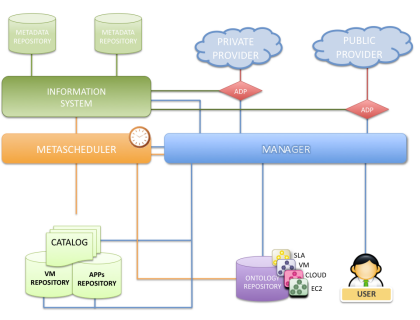

Figure 1. System architecture overview

the components responsible for abstracting the APIs of the different providers and expose a set of functionalities to our system. They are also responsible for generating all the information about the resources of the providers and storing it on the Information System

\section{Semantic Model}

To provide a framework for handling all the information about the workflow, resources and processes involved in the definition of the virtualized infrastructure, we propose a Semantic Model (depicted in figure 2) comprising a set of semantic technologies.

As part of our architecture we define an Ontology Repository in which all the models for describing the whole system are stored. These models describe the relevant aspects of each providers and also the relations en equivalences between them. They also describe all the aspects of the workflows and their requirements and map them to the related concepts of our system.

We also propose the use of Linked Data technologies as a light-weight RDF system to manage all the information about the system. We argue that describing and linking several providers and virtual machine images catalogs will improve the outcome of our system.

As part of this Semantic Model we propose a set of features:

- Reasoning: based on the models and the data generated based on them we want to explore how a reasoning process could infer new useful data about the system.

- Optimization: to minimize the size of the virtualized infrastructure and hence reducing it cost.

- Validation: to ensure that the requirements of the workflow can be satisfied and its description is consistent.

- Resource discovery: we wan to explore how Linked Data and link discovery techniques could improve the resource discovery process of our system.

- Collaborative Annotation: bringing the final users of the system to add and describe resources of the system.

\section{Hypothesis, RESTRICTIONS AND AsSUMPTIONS}

To formalize all the ideas exposed above we introduce the main hypothesis of this thesis and the facts (assumptions) and limitations (restrictions) considered for this work. 


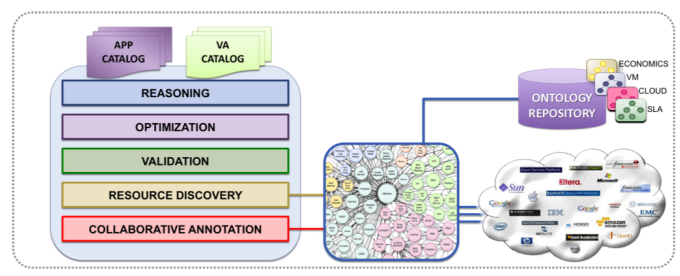

Figure 2. Semantic Model overview

\section{A. Hypothesis}

Semantics allow to solve interoperability and heterogeneity problems in information management and decision making processes for different models, providers and solutions for virtualized infrastructures in the context of scientific workflows, taking into account the Quality of Service exposed and required by the different parts involved.

\section{B. Assumptions \& Restrictions}

- Assumption 1: There are several solutions and providers for virtualizing computing infrastructures, each one of them exposing its services through their own APIs and services, and using its own vocabularies.

- Assumption 2: The sets of applications and tools used in the scientific workflows belonging to the same scientific area are frequently the same and can be reused among those workflows.

- Assumption 3: Semantics technologies are expressive enough to describe applications used in scientific workflows, their dependencies, configurations and requirements.

- Assumption 4: Reproducibility is more important than performance in a scientific experiment. That is, it is more important to replicate the same results than improving the time to obtain them.

- Restriction 1: As we deal with external providers and solutions, each one of them defining its own QoS, we can not offer a custom QoS or SLA. If a user requires an execution that exceeds the QoS offered by those providers his/her workflow will not be able to be executed

- Restriction 2: We can not guarantee that we will generate an infrastructure configuration that ensures the execution to be performed in a certain amount of time. That is, we can guarantee that we every time we run the experiment we will obtain the same results, but the processing time may vary from one execution to another.

- Restriction 3: Considering the whole spectrum of possible applications and versions that could be used in a workflow is not feasible and therefore we assume the subset of the most common ones for the workflows belonging to a concrete area of science.

\section{EXPECTED OUTCOME}

Based on the ideas exposed throughout this paper we expect to obtain as a result of this thesis the following:

- An architecture that allows to integrate a workflow management system with a set of virtualized infrastructure providers.

- A semantic model expressive enough to describe all the components of each provider, the workflow software and hardware requirements and the set of policies considered for generating the infrastructure configuration.

- A system able to generate, based on the information retrieved from providers and the description of the workflow, a configuration defining the set of computational resources that must be run and the software stack that must be deployed on each one of them.

- A proof-of-concept implementation of the system that will work with a set of providers that allow us to verify that it is possible to generate the infrastructure configuration and to evaluate the performance of the system.

\section{CONCLUSIONS}

In this paper we propose a set of ideas that fill the gap between scientific workflows and their target infrastructure by enhancing the workflow description and using it to provide a more dynamic, optimal and replicable infrastructure.

This work is in its early stages and in the next months we plan to refine the assumptions and restrictions of the thesis and also define a set of concrete scenarios in which it could be applied to test its feasibility.

\section{REFERENCES}

[1] P. Haase, T. Mathäß, M. Schmidt, A. Eberhart, and U. Walther, "Semantic technologies for enterprise cloud management," in International Semantic Web Conference (2), 2010, pp. 98-113.

[2] S. Haak and S. Grimm, "Towards custom cloud services - using semantic technology to optimize resource configuration," in $E S W C$ (2), 2011, pp. 345-359.

[3] A. V. Dastjerdi, S. G. H. Tabatabaei, and R. Buyya, "An effective architecture for automated appliance management system applying ontology-based cloud discovery," in Cluster, Cloud and Grid Computing (CCGrid), 2010 10th IEEE/ACM International Conference on, may 2010, pp. $104-112$.

[4] "Open virtualization format," 2012. [Online]. Available: http://www.dmtf.org/standards/ovf

[5] "mosaic: D1.1: Architectural design of mosaics api and platform," Feb. 2011. [Online]. Available: http://www.mosaiccloud.eu/dissemination/deliverables/FP7-256910-D1.1-1.0.pdf

[6] "Amazon Web Services." [Online]. Available: http://aws.amazon.com

[7] "Aws marketplace," 2012. [Online]. Available: https://aws.amazon.com/marketplace

[8] "Aws simple workflow service," 2012. [Online]. Available: https://aws.amazon.com/swf

[9] "Pegasus workflow management system." [Online]. Available: http://pegasus.isi.edu/

[10] "Taverna workflow management system." [Online]. Available: http://www.taverna.org.uk/

[11] "Wings: Workflow instance generation and selection." [Online]. Available: http://wings.isi.edu/ 